\title{
Changes in Neural Activation Patterns and Brain Anatomy as a Function of Non- Pathological First Language Attrition
}

\section{Merel Keijzer}

Center for Language and Cognition, University of Groningen, The Netherlands

*Corresponding author: Merel Keijzer, Center for Language and Cognition, University of Groningen, Oude Kijk in't Jatstraat 26 , 9712 EK Groningen, The Netherlands, Tel: + 31-50-363-7273, Fax: + 31-50-363-5821; E-mail: m.c.j.keijzer@rug.nl

Rec date: May 23, 2014, Acc date: Jul 23, 2014, Pub date: Jul 27, 2014

Copyright: (C) 2014 Keijzer M. This is an open-access article distributed under the terms of the Creative Commons Attribution License, which permits unrestricted use, distribution, and reproduction in any medium, provided the original author and source are credited.

\begin{abstract}
In recent years the Critical Period Hypothesis of language acquisition has come under close scrutiny. The premise that native-like language proficiency can only be attained if the language is learned early in life seems difficult to maintain, as neuroimaging data have revealed a greater plasticity of the human brain to master new languages than has previously been assumed. The field of non-pathological first language (L1) attrition has not contributed much to this debate. Adding attrition perspectives, however, can inform the field of language learning and the critical period in general: learning a language early in life should leave long-lasting traces in the neural circuit. But investigations of this nature would also directly benefit the field of $L 1$ attrition itself. Attrition theories have largely built on behavioral paradigms, and two pivotal questions remain unanswered but could be addressed using neuroimaging techniques: 1 ) Is the cause of L1 attrition mainly L1 non-use or rather the introduction and mastery of a second language (L2)? 2) is L1 attrition an irreversible, permanent phenomenon or does it merely reflect a temporary inaccessibility of the L1 system? This paper aims to review the scantly available evidence for functional and/or anatomical brain changes as a function of non-pathological L1 attrition, specifically focusing on the two outstanding questions above. Building on previous insights, this paper theorizes about L1 attrition-induced neurological changes that have not been addressed in previous work and formulates goals and avenues for future studies.
\end{abstract}

Keywords: Non-pathological first language attrition; Neural activation changes; Brain anatomy changes; Temporary versus permanent loss; L1 non-use versus L2 interference

\section{Introduction}

Until only recently, a prevalent view in the field of language acquisition, most notably second language (L2) acquisition, was that language had to be learned before a certain age in order for the speaker to function at a native-like level. This theory, referred to as the Critical Period Hypothesis [1], builds on the premise that the human brain loses its plasticity to learn language at roughly the onset of puberty and uses as its main source of evidence the differences attested in both learning trajectories and outcomes of child and adult second language learners [2]. In more recent years, however, the theory has been modified and age bands extended to accommodate cases of people who attain native-like proficiency levels in their L2, despite learning it later in life. Behavioral evidence of this kind has thus caused the critical period to come under scrutiny. (cf. [3] for a discussion). Even more compelling evidence refuting the Critical Period Hypothesis comes from neurocognitive studies that have shown the language function of the human brain to be much more flexible than has previously been assumed. This is in line with what we know about neuroplasticity in other domains [4]. Because of advances in neuroimaging techniques, such as the Event-related potential (ERP) technique and (functional) Magnetic Resonance Imaging ((f) MRI), it has been convincingly shown that even the brains of speakers who learn a second language (L2) later in life can resemble those of mother tongue speakers (L1) in their functional activation patterns, given sufficient, consistent and long-term exposure to the L2 [4]. More converging evidence comes from structural MRI scans, which reveal that such long-term L2 activation can even lead to anatomical brain changes, most notably in the realms of increased gray matter density, white matter integrity and cortical thickness [(see [4] for an overview of previous work in this area).

What is counterintuitive is that almost no attention has been paid to the other side of the coin: what happens to the neurological organization of the first language following long-term L2 exposure? From the language pathology literature, we know that changes in brain organization following a stroke or brain bleed may-depending on the lesion site-heavily impact on language and can result in aphasia or aphasic symptoms [5]. Aphasic recovery patterns point to the brain's plasticity and ability to rewire, which is especially interesting to observe in the case of bilingual patients: mostly, a patient's languages have been found to recover in parallel. However-in part depending on pre-lesion proficiency levels-asymmetric recovery patterns have also been reported [6,7]. But non-pathological language attrition is markedly different from pathological language loss such as aphasia. Most commonly attested in immigrant populations who left their L1 environment and are now immersed in an L2 setting, non-pathological language attrition is gradual as opposed to sudden [8]. Perhaps most importantly, rather than anatomical and functional brain changes leading to language change in the case of pathological loss, nonpathological attrition operates the other way around: here changing language use and dominance patterns may give rise to changing functional and anatomical brain organization. Given the available evidence from the related field of second language acquisition, it seems plausible to postulate that long-term L2 use and L1 non-use lead to both functional and anatomical brain changes. After all, on a 
behavioural level, rather severe L1 changes have been reported following a prolonged period of immersion in an L2 environment [9]. However, the neural correlates of changes in behavioral performance remain unclear; neuroimaging studies within the field of nonpathological L1 attrition are extremely scarce. Because of that, two questions that have dominated the field since its inauguration remain unanswered: 1) Is it mainly L2 use or reduced L1 use that ultimately leads to attrition? 2) Is the attrition that speakers experience permanent or does it merely reflect a temporary inaccessibility of the L1 system?

This paper aims to review the scantly available evidence for functional and/or anatomical brain changes as a function of nonpathological L1 attrition, specifically focusing on the outstanding questions above. Building on previous insights explicated in section 2 and section 3 theorizes about L1 attrition-induced neurological changes that have not been addressed in previous work and formulates goals and avenues for future studies. A better understanding of the neurological consequences of non-pathological language attrition can provide insights into the dynamic neural organization of language and, ultimately, the neuroplasticity of the brain as a whole.

\section{The Changing Brains of L1 Attriters: What We Know from Previous Work}

With its inauguration some 40 years ago, the field of L1 attrition has become firmly embedded in the larger research realm of bilingualism. The now rich behavioral literature indicates that the speech of L1 attriters immersed in an L2 environment does undergo changes, although not all domains are affected as much and as fast. The lexicon has been identified as most susceptible to attrition, with the grammatical and phonological domain appearing more robust to changes $[9,10]$. But since adult immigrants very rarely return to their L1 environment, past work has not been able to disentangle these effects as (temporary) changes in L1 processing or (more permanent) changes in L1 representations [11]. propose that bilingualism does have behavioural and neurological consequences for language processing, including L1 processing, but only in extreme circumstances does this lead to L1 attrition of representations. By contrast, evidence from the related field of second language acquisition suggests that the most successful L2 learners are those who tolerate changes to their L1 and this effect is especially noticeable in the initial stages of mastering the L2 because it is then that the L1 needs to be most strongly inhibited to avoid interference [12]. This is underscored by the robust psycholinguistic finding that the costs of switching from the L2 to the L1 are far greater than vice versa [13], and is also in line with reports that most attrition occurs within 5 years of the onset of L2 immersion [14]. The only way to resolve this issue is by using a (semi)-longitudinal design to collect neuroimaging evidence which not only shows different functional activation patterns in L1 processing in those speakers who are (recently) immersed in an L2 environment, but also how long-term L2 immersion may eventually lead to anatomical differences in the brains of L1 attriters when compared to speakers in the country of origin.

To date, only a handful of neuroimaging studies have examined the neural representation of the L1 in L2 immersed subjects. Most iconic perhaps is the work by Christophe Pallier et al. [15], who looked at 8 Korean subjects who were adopted into Francophone families between the ages of 3 to 8 . Now in their mid to late twenties, the subjects reported no recollection of the Korean language, which was corroborated by behavioural tests. More importantly, event-related
fMRI experiments revealed no specific cortical activation in these subjects when listening to Korean stimuli. They were, in fact, no different from a group of native French controls. When being tested using French stimuli, the adoptees showed similar activated brain areas as the French controls, although the activation site for the French natives was more extensive. The authors use this finding as converging evidence against the existence of a critical period, as learning a language early in life should leave long-lasting traces in the neural circuit, but instead French here appears to have 'overridden' Korean in the adoptees' brains [16]. Using more fine-grained follow-up tests of, among others, a recognition number series, the investigators were able to differentiate between phonological and semantic memory, but only minimally, claiming that "the adoptees have a somewhat more precise notion of the sound pattern of Korean than the native French, but no explicit access to knowledge of Korean lexical items" [16, p. 219]. Anecdotal reports of retraining in this subject pool, where previous experience with Korean should facilitate learning the language, did not amount in significant findings either: many of the adoptees visited Korea (with visits ranging from a few days to a few months) and/or took Korean language courses. None showed a significant recovery of Korean. In short, despite substantial early-life exposure to Korean, virtually no traces of the language were found in this group of international adoptees. What is not considered in Pallier's work, however, is that traumatic childhood experiences, of which international adoption may certainly be considered one, has been shown to alter neurological organization patterns [17]. In addition, the structural MRI scans that were collected from the adoptees as part of Pallier's fMRI study were not compared on anatomical differences with those of the French natives, which may have rendered interesting differences.

To date, no whole brain (f) MRI work has been done on L1 speakers immersed in an L2 environment who left their home country as adults and by their own choice. However, such speakers have been looked at using Event-related potential (ERP) techniques and eyetracking measures. ERP measures record voltage changes in the brain following stimulus presentation in a time-locked manner [18]. Past ERP investigations of $\mathrm{L} 1$ attrition have looked at, for instance, grammatical gender in L1 German attriters and found that, overall, this group still processed grammatical gender violations in a native-like manner. More specifically, P600 effects were elicited upon encountering an ungrammatical German article choice, which is commonly attested when native speakers come across a grammatical violation. However, the subjects were found to have a different scalp distribution of this effect than a German control group in the home country [19]. Diverging evidence is found in Dussias [20], who used an eyetracking paradigm to look at processing of complex Noun Phrases (NPs) by native L1 Spanish speakers with prolonged L2 English immersion experience. The eye-gaze patterns of these speakers indicated that they attach relative clauses to the second verb in a complex NP, as is common in English but not in Spanish (where the attachment is predominantly a non-local one, and the relative clause is attached to the first noun instead). Follow-up research suggested that this finding, where the L1 is essentially processed as if it were the L2, is already attested in speakers with minimal L2 immersion experience [21]. In other words, within the grammatical domain, the only evidence for restructuring comes from eyetracking evidence, which is generally considered a behavioral measure rather than a neuroimaging technique.

With regard to the open attrition questions, the strong L2 transfer effect attested in Dussias' work points towards L2 influence as the 
cause for attrition, rather than L1 non-use. On the basis of these findings from the grammatical domain, no conclusive evidence can be presented vis-à-vis the irreversibility of L1 attrition, however.

Apart from the grammatical domain, ERP work has also been done in the area for which most dramatic losses are typically reported in L1 attrition: the lexicon. Datta tested 27 L1 Bengali speakers immersed in the US, with an age range between 10 to 62 years old, in a cross-modal and cross-linguistic design: subjects saw a picture (pre-identified as highly or lowly familiar in either Bengali or English, or both) and were asked to implicitly name the picture in whatever language (L1 Bengali or L2 English) came to mind [22]. They then heard an audio recording of the word, in either Bengali or English. Their ERP waveforms were recorded as well as a behavioural response where subjects were asked to provide a binary button press: ' 1 ' if the word had one syllable and ' 5 ' if the word had more syllables. This set-up elicited longer RTs for Bengali in cases where the word was highly familiar in both languages and longest RTs for English in cases where words were highly familiar in Bengali but not in English. In addition, the ERP signatures showed a more negative deflation to English and Bengali words that were relatively unfamiliar in English, regardless of their Bengali ratings. There was, moreover, a differential pattern for L2 or L1 dominant participants, leading to the overall conclusion that the dominant cause of L1 attrition is L2 interference, but only in L2 dominant individuals. Similar to ERP work examining the grammatical system, this lexical study's findings do not allow insights into the temporary versus permanent nature of attrition.

Overall, the scantly available evidence to date is very inconsistent when it comes to changes in neural activation and organization in L1 attriters and often fails to find any evidence altogether. That could indicate that such reorganization simply does not occur the way it has been reported for second language acquisition, but is not in line with what we know from L2 learning. In fact, the available evidence uniformly points to $\mathrm{L} 2$ interference as the main cause of attrition rather than L1 non-use. In other words, prolonged L2 use has an effect on brain activation and organization patterns, and cascades down to the L1. This ties in with findings from the general learning and memory literature; retrieval-induced forgetting theories, for instance, propose that newly acquired information can most definitely interfere with older memory traces [23]. This finding in turn has triggered studies showing that naming (words) in the L2 can hinder subsequent L1 retrieval $[12,24]$. Similarly, the theory of catastrophic interference whereby the sudden introduction of new information may have a deep impact on earlier stored representations [25] - has been invoked in the context of L2 acquisition and subsequent L1 attrition: with the advent of an L2, L1 representations are likely to change [26].

Behavioral attrition work has shown different language domains to be affected at different times. Most notably, the lexicon has been shown to be most and earliest affected and domains like phonology have been found more resilient to changes. This is mostly explained on the basis of regression-type arguments [10,27]: phonological encoding tends to precede lexical acquisition in children and is therefore better entrenched, making it resilient to loss. The scantly available neuroimaging for attrition also suggests that phonology is more spared than the lexicon: [16] differentiate between phonological and semantic memory in that their adoptees do have a somewhat clearer notion of the Korean sound pattern than the native French controls, but no recollection of Korean lexical labels. The binary phonology-semantics distinction also features prominently in the retrieval induced forgetting literature, where differential effects have consistently been found for semantic and phonological representations. As opposed to the pattern found in [16], however, the hierarchy operates in reverse order in the retrieval induced forgetting literature: introducing new materials generally only has a detrimental effect on recalling phonological information and tends to spare-and even facilitateretrieval of L1 semantic information. In other words, when L1 attriters are asked to name as many L1 exemplars as possible belonging to a certain semantic category (like animals) in a limited time span, they tend to perform on a par with non-attriting peers. It is when they are asked to generate items starting with a certain letter, however, that discrepancies between the two groups start to occur. More converging evidence for the semantics-phonology dissociation comes from work on age-related decline of language functions. Semantic processes are well preserved in older adulthood, which may be surprising given the known gray and white matter reductions as a function of age. By contrast, elderly speakers do show marked difficulties with phonological retrieval, evidenced in more slips of the tongue [28]. This asymmetric pattern between phonological and semantic processes thus suggests that the two are dissociable. This makes them ideally suitable as a testing ground to study functional activation brain patterns as a result of L1 attrition. Such a comparison between semantic and phonological attrition is all the more interesting given the discrepancies between behavioral and neuroimaging findings in spared versus affected domains in attrition. From the available evidence it seems that phonological retrieval appears more the result of L2 interference while failure to retrieve L1 lexical items more directly follows from not using the L1 as regularly anymore.

\section{The Changing Brains of L1 Attriters: What We Need To Learn from Future Studies}

In the realm of $\mathrm{L} 2$ acquisition, neuroimaging methods have shown complementary patterns of functional and anatomical brain changes as a function of time: the cumulative efforts of past ERP research has shown that the brain outpaces behavior: even when L1 dominant bilinguals in the initial stages of L2 acquisition perform at chance level in their L2, their brain waves already tend to look quite different from those of monolingual controls [29]. And although functional Magnetic Imaging Resonance data have collaborated this finding, anatomical changes, assessed through structural MRI scans, are not brought about quickly and only follow prolonged periods of consistent L2 exposure. Even when change does occur, anatomical structure changes in isolated brain areas do not necessarily imply that the subject is a (near)-native speaker of a language [4]. Past second language studies have commented on the dynamic interaction between the L1 and L2, with long-term L2 exposure having repercussions for the L1 on a functional brain activation as well as anatomical level [4], but the question how long and consistent the L2 exposure needs to be in order for functional changes to settle into irreversible anatomical adaptions is not met by neuroimaging data in the field of $\mathrm{L} 1$ attrition.

To answer the two outstanding attrition questions regarding the cause and irreversible nature of non-pathological L1 loss, it is imperative to accumulate more neuroimaging data, preferably combining behavioral, ERP and (f) MRI methods. The hierarchical order of attested changes in L2 learning (first observed in ERP waveforms and functional brain activation patterns before showing up as anatomical and/or behavioral changes) likely extends to L1 attrition and directly relates to the question of temporary versus permanent changes to the L1 system: earlier ERP waveform and functional brain activation changes most likely reflect a temporary state of bilingual 
language processing, even though - on a behavioral level-émigrés still perform on a par with native speakers in the home language environment. Anatomical changes, underscored by changed behavioral patterns, are then likely indicators of more permanent L1 loss. In order for neuroimaging data to be truly meaningful, however, a number of focus points need to form the basis for future research endeavours.

Given the mixed findings from previous L1 attrition ERP work and the general poor spatial resolution of this method, (f) MRI scans bear most potential in answering the remaining attrition questions. As no studies currently exist in this field other than the work of Christophe Pallier and his colleagues, all fMRI investigations-ideally mirroring ERP research designs-are informative. In line with Pallier's design and in closely resembling ERP paradigms, event-related fMRI measures seem more appropriate than blocked designs, as this method can more directly contrast subjects' responses to two different language systems. Additionally, in recent years, resting-state fMRI measures have received more attention. The traditional fMRI technique relies on task-based performance and measures cerebral blood flow changes correlated with neural task-related activity through the Blood Oxygen Level Dependent (or BOLD) contrast, which maps the vascular system's response to the active brain regions' metabolic need for more glucose [32]. Recently, however, fMRI work has built on the discovery that when subjects lie awake in the scanner but do not carry out any particular tasks (i.e. are at rest), a low-frequency BOLD signal can be detected that is indicative of a coherent activity within distinct functional networks, including the language system $[33,34]$. This spontaneous low-frequency fluctuation (also referred to as LFF) of the BOLD signal is hypothesized to reflect unconstrained cognitive processing during resting states [34], and results in an image of the socalled default network. Default networks have been shown to be dynamic (changing as a function of age, for instance) and their functional role is generally considered to be the maintenance and coordination of neuronal activity that underlies everyday task activation, so default networks could be considered a memory consolidation device of task activities during the day. It is pivotal to assess if the default networks of long-term residents of an L2 environment show marked differences compared to L1 speakers in the home language environment, as actively using the L1 is no longer part of attriters' daily tasks.

Similarly, fMRI investigations-by default-collect structural, T1 weighted, MRI scans to co-register the functional scans of interest, but only in until recent years have such anatomical scans themselves become the main focus of interest. In the language science structural MRI scans are examined in an attempt to uncover anatomical changes in long-term consistent L2 users [4]. Anatomical changes of this nature have been attested in L2 speakers who do or do not live immersed in an L2 environment. Within the latter category, it becomes hard to disentangle anatomical change due to reduced L1 use or increased L2 use, while crucial for our understanding of the phenomenon of L1 attrition. To bypass this problem, a possible research avenue for future studies would be to contrast the T1 weighted images of long-term L2 immersed subjects with those of monolingual speakers of the L1 and L2, but also include a group of advanced L2 speakers in a non L2 environment. This latter combination is commonly applied in attrition work [35], but not using neuroimaging techniques. To simultaneously address the questions of cause and irreversible nature of attrition, it is equally important to use semi-longitudinal designs where subjects are followed over the course of several years (as a yardstick, a timespan of 3 years is sufficient and also makes such an endeavor feasible within a given research project span). Furthermore, any such design should target subjects with different lengths of residence in the L2 environment cross-sectionally, starting from 0 years through to 10 years abroad (which is commonly employed as a lower threshold in behavioral attrition work). Such a design allows for a detailed investigation of the 'turning point' of L1 processing and representation: when do speakers start processing their L1 as if it is their L2 and when-if ever-does this change lead to anatomical brain adaptations?

One methodological difficulty that has dominated attrition research is the intersubject variability. Given the same extent of exposure and L1 use patterns, some individuals-on behavioral level-show substantial deviances from the native norm while the speech of others remains virtually unaffected. This has been ascribed to interlearner differences in cognitive resources, irrespective of language experience [36]. The phenomenon of individual differences is not unique to L1 attrition and has been widely reported in the L2 learning literature, but in that field is partly collaborated by neuroimaging evidence [4] cite a number of functional MRI studies that have explored the issue of individual differences in mastering a new language. What those studies have collectively shown is that functional neural patterns in L2 learners can and do capture differences in learning, and resting-state functional connectivity work can even be used to predict such differences [37]. This line of work can easily be extended to L1 attrition contexts, allowing a closer inspection of the individual differences and using these as predictors of the severity of attrition that characterizes any person's L1 system. This constitutes a welcome change to the stance towards individual differences currently taken in attrition research, where they are seen as problematic rather than informative.

With the inability of the Critical Period Hypothesis to explain data patterns on a behavioral and neuroanatomical level, several accounts have been formulated to alternatively capture the L2 learning process and the brain's flexibility to deal with new languages as well as with individual differences attested. A strong model in this regard is the competition model. First formulated in the late 80s [38], and having gone through a series of reformulations and refinements [39], the model emphasizes the dynamic nature of L1 and L2 interactions, where changes that are brought about are both the result of input (experience) and synaptic adaptations and are constantly being governed by constructs like competition, entrenchment, selforganization, local and global reorganization and Hebbian learning [4]. According to this model, L1 attrition would then not be caused either by L1 non-use of L2 mastery, but is rather an interaction between the two. One undoubtedly impacts on the other, as previously suggested by [40], who claimed that attrition results from L2 interference, accelerated by not using the $\mathrm{L} 1$ daily anymore, but future work still needs to shed more light on the contributions of each model, ideally following the guidelines set out above.

Different language domains are known to behave differently, as shown by past behavioral attrition work as well as behavioral and neurocognitive L2 investigations. It may therefore be most informative to focus on one language domain for an initial neurocognitive exploration of L1 attrition. Given that the lexicon is the most and earliest affected domain in L1 attrition, this would be the best candidate, especially as this domain allows for the introduction of various levels of complexity, informed by previous findings. Most importantly, the dissociation between phonological and semantic learning and memory consolidation that has been found in past behavioral investigations [12] as well as in past fMRI work (albeit in 
the field of language and cognitive aging in monolinguals; [28]) can reveal if the same (lexical) domain is further subdivided (in phonology and semantic components) regarding the timeframe in which functional and/or anatomical changes in the brains of L1 attriters manifest themselves. Additionally, it allows for a controlled introduction of effects that have been found to influence bilingual processing in the past: cognate versus non-cognate status of words and frequency effects may all impact on a word's retention and attrition. This can get at the question of L2 transfer effects or L1 internal restructuring effects causing attition, leading to more insights into the ultimate cause of $\mathrm{L} 1$ attrition.

Thus bringing together past studies' evidence-both behavioral and that obtained from neuroimaging techniques-of L1 attrition but also of the related and much more explored field of L2 learning, several hypotheses can be formulated with respect to L1 lexical attrition, to be empirically assessed by future studies. Three such questions/ hypothesis that appear in most dire need of further investigations are:

Non-pathological L1 attrition is a two-step hierarchical phenomenon: changed functional activation patterns, assessed through ERP and fMRI methods, are revealed (very) soon following L2 immersion, although subject to individual differences those characterize especially the initial stages of $\mathrm{L} 1$ attrition.

As L2 proficiency increases, theories such as retrieval induced forgetting, would predict that speakers can relax more vis-à-vis the inhibition of their L1. In other word, the functional processing differences witnessed in the initial stages of L2 immersion may level out. At the same time, prolonged L2 immersion leaves its mark: whereas the temporary processing differences may fade away, anatomical structural changes are revealed at later stages of attrition. Being much more subtle in nature than the initial processing changes, structural anatomical changes as a function of L1 attrition are not witnessed for all speakers across the board, but for those subjects in whom anatomical changes do appear, they are irreversible.

The dynamic interaction between the L1 and the L2 is different for different language domains and subdomains. Within the lexicon, for instance, phonological memories are more prone to $\mathrm{L} 2$ influence than semantic representations. That is not to say that semantic representations are not affected, but failure to retrieve an L1 lexical item follows more from L1 non-use than L2 interference.

\section{Conclusion}

Although in recent years the critical period of language acquisition has come under close scrutiny as a direct consequence of neuroimaging evidence revealing a greater plasticity of the human brain to master new languages than has previously been assumed, the field of non-pathological L1 attrition has not contributed much to this debate. Not only would this be informative for the field of language learning and the critical period in general, as learning a language early in life should leave long-lasting traces in the neural circuit, but investigations of this nature would also directly benefit the field of L1 attrition. As most attrition theories have been formulated on the basis of behavioral evidence, two pivotal questions that have dominated the field since its inauguration remain unanswered: 1) Is the cause of L1 attrition mainly a reduction in L1 use patterns or rather the introduction and mastery of a second language, and 2) Is L1 attrition an irreversible, permanent phenomenon or does it merely reflect a temporary inaccessibility of the L1 system? This paper has reviewed the scantly available neuroimaging evidence from the field of L1 attrition as well as the much richer literature of second language learning to investigate why these two questions remain as yet unanswered. In addition, this paper has theorized about the nature of L1 attrition based on what we do know. It has attempted to identify caveats for future (neuroimaging) studies to explore. The main argument put forward in this paper is that L1 attrition is a two-step phenomenon whereby functional brain activation patterns precede structural anatomical differences that are furthermore highly subjected to individual differences. These individual differences themselves are the interplay between external experience-driven change and internal (neural) restructuring.

\section{Acknowledgments}

The idea for this paper was inspired by stimulating discussions within the lab meetings of Prof.dr. Janet van Hell's Bilingualism and Language Develoment (BiLD) Lab at The Pennsylvania State University. The author would like to acknowledge the contributions of all lab members, most notably Kaitlyn Litcofsky whose co-authored paper (with Ping Li and Jennifer Legault) formed the direct impetus for this review. Preparation of this manuscript was made possible by the Netherlands Organisation for Scientific Research (NWO) Veni Innovation Scheme Grant 275-70-030 to Merel C.J. Keijzer, which is hereby gratefully acknowledged.

\section{References}

1. Lenneberg EH (1967) Biological foundations of language. Wiley, New York, USA.

2. DeKeyser R (2000) The robustness of critical period effects in second language acquisition. Studies in Second Language Acquisition 22: 499-533.

3. Rothman J (2008) Why all counter-evidence to the Critical Period Hypothesis in second language acquisition is not equal or problematic. Language and Linguistics Compass 2: 1063-1088.

4. Li P, Legault J, Litcofsky KA (2014) Neuroplasticity as a function of second language learning: Anatomical changes in the human brain. Cortex .

5. Kolk HHJ (2006) How language adapts to the brain. In Progovac L, Paesani K, Gasielles E, Barton E. (Eds.) The syntax of nonsententials: Multi-disciplinary perspectives. John Benjamins Publishing Company, Amsterdam, the Netherlands.

6. Paradis M (2001) (Ed.) Manifestations of aphasia symptoms in different languages. Pergamon Press, Oxford, UK.

7. Glitterman MR, Goral M, Obler LK (2012) Aspects of multilingual aphasia. Multilingual Matters, Bristol, UK.

8. De Bot K, Weltens B (1991) Recapitulation, regression and language loss. In Seliger H, Vago RM (Eds.) First language attrition: Structural and theoretical perspectives. Cambridge University Press, Cambridge, UK.

9. Schmid MS (2013) Language attrition. Cambridge University Press, Cambridge, UK.

10. Keijzer MCJ (2007) Last in first out? An investigation of the regression hypothesis in Dutch emigrants in Anglophone Canada. LOT Publications, Utrecht, the Netherlands.

11. Costa A, Sebastián-Gallés N (2014) How does the bilingual experience sculpt the brain? Nat Rev Neurosci 15: 336-345.

12. Linck JA, Kroll JF, Sunderman G (2009) Losing access to the native language while immersed in a second language: evidence for the role of inhibition in second-language learning. Psychol Sci 20: 1507-1515.

13. Bobb SC, Wodniecka Z (2013) Language switching in picture naming: what asymmetric switching costs (do not) tell us about inhibition in bilingual speech planning. Journal of Cognitive Psychology 25: 568-585. 
Citation: Keijzer M (2014) Changes in Neural Activation Patterns and Brain Anatomy as a Function of Non-Pathological First Language Attrition.

Page 6 of 6

14. Hutz M (2004) Is there a natural process of decay? In Schmid MS, Köpke B, Keijzer M, Weilemar, L. (Eds.) First language attrition: Interdisciplinary perspectives on methodological issues. John Benjamins Publishing Company, Amsterdam, the Netherlands.

15. Pallier C, Dehaene S, Poline JB, LeBihan D, Argenti AM, et al. (2003) Brain imaging of language plasticity in adopted adults: can a second language replace the first? Cereb Cortex 13: 155-161.

16. Ventureyra V, Pallier C, Yoo, Y-H (2004) The loss of first language phonetic perception in adopted Koreans. Journal of Neurolinguistics 17: 79-91.

17. Perry BD, Pollard RA, Blaicley TL, Baker WL, Vigilante D (1995) Childhood trauma, the neurobiology of adaptation, and "use-dependent" development of the brain: how "states" become "traits". Infant Mental Health Journal 16.4: 271-291.

18. Luck SJ (2005) An introduction to the event-related potential technique. MIT Press, Cambridge, USA.

19. Loerts H, Stowe LA, Schmid MS (2013) Predictability speeds up the reanalysis process: an ERP investigation of gender agreement and cloze probability. Journal of Neurolinguistics 26: 561-580.

20. Dussias PE (2003) Syntactic ambiguity resolution in L2 learners: some effects of bilinguality on L1 and L2 processing strategies. Studies in Second Language Acquisition 25: 529-557.

21. Dussias PE (2004) Parsing a first language like a second: the erosion of L1 parsing strategies in Spanish-English bilinguals. International Journal of Bilingualism 3: 355-371.

22. Datta H (2010) Brain bases for first language lexical attrition in BengaliEnglish speakers. Doctoral Dissertation, The City University of New York, USA.

23. Anderson MC, Spellman BA (1995) On the status of inhibitory mechanisms in cognition: memory retrieval as a model case. Psychol Rev 102: 68-100.

24. Levy BJ, McVeigh ND, Marful A, Anderson MC (2007) Inhibiting your native language: the role of retrieval-induced forgetting during secondlanguage acquisition. Psychol Sci 18: 29-34.

25. McClosky M \& Cohen N (1989) Catastrophic interference in connectionist networks: the sequential learning problem. In Bower GH (Ed.) The psychology of learning and motivation. Academic Press.

26. Bakker I, Takashima A, van Hell JG, Janzen G, McQueen JM (2014) Competition from unseen or unheard novel words: lexical consolidation across modalities. Journal of Memory and Language 73: 116-130.
27. Jakobson R (1941) Kindersprache, Aphasie und Allgemeine Lautgesetze. Almqvist, Uppsala, Sweden.

28. Diaz MT, Johnson MA, Burke DM, Madden DJ (2014) Age-related Differences in the Neural Bases of Phonological and Semantic Processes. J Cogn Neurosci .

29. McLaughlin J, Osterhout L, Kim A (2004) Neural correlates of secondlanguage word learning: minimal instruction produces rapid change. Nat Neurosci 7: 703-704.

30. Luk G, Green DW, Abutalebi J, Grady C (2011) Cognitive control for language switching in bilinguals: A quantitative meta-analysis of functional neuroimaging studies. Lang Cogn Process 27: 1479-1488.

31. Andrews E, Frigau L, Voyvodic-Casabo C, Voyvodic J, Wright J (2013) Multilingualism and fMRI: Longitudinal Study of Second Language Acquisition. Brain Sci 3: 849-876.

32. Buxton R (2009) Introduction to functional magnetic resonance imaging principles and techniques. Cambridge University Press, Cambridge, UK.

33. Binder JR, Frost JA, Hammeke TA, Bellgowan PS, Rao SM, et al. (1999) Conceptual processing during the conscious resting state. A functional MRI study. J Cogn Neurosci 11: 80-95.

34. Tie Y, Rigolo L, Norton IH, Huang RY, Wu W, et al. (2014) Defining language networks from resting-state fMRI for surgical planning--a feasibility study. Hum Brain Mapp 35: 1018-1030.

35. Hopp H, Schmid MS (2013) Perceived foreign accent in L1 attrition and L2 acquisition: the impact of age of acquisition and bilingualism. Applied Psycholinguistics 34(2): 361-394.

36. Keijzer M (2013) Working memory capacity, inhibitory control and the role of 12 proficiency in aging 11 dutch speakers of near-native 12 english. Brain Sci 3: 1261-1281.

37. Ventura-Campos N, Sanjuán A, González J, Palomar-García MÁ, Rodríguez-Pujadas A, et al. (2013) Spontaneous brain activity predicts learning ability of foreign sounds. J Neurosci 33: 9295-9305.

38. Bates E, MacWhinney B (1987) Competition, variation and language learning. In MacWhinney B (Ed.) Mechanisms of language acquisition. Lawrence Erlbaum, Hillsdale, USA.

39. Li P, MacWhinney B (2013) Competition Model. In Chapelle CA (Ed.) The encyclopedia of applied linguistics. John Wiley and Sons, Malden, USA.

40. Silva-Corvalàn C (1994) Language contact and change. Oxford University Press, Oxford, UK. 